\title{
Application of a Home Energy Management System for Incentive-Based Demand Response Program Implementation
}

\author{
Omid Abrishambaf ${ }^{1}$, Mohammad Ali Fotouhi Ghazvini ${ }^{1}$, Luis Gomes ${ }^{1}$, Pedro Faria ${ }^{1}$, Zita Vale ${ }^{1}$, J. M. Corchado ${ }^{2}$ \\ ${ }^{1}$ GECAD - Knowledge Engineering and Decision Support Research Centre, Polytechnic of Porto (IPP), Porto, Portugal \\ ${ }^{2}$ BISITE Research Centre, University of Salamanca, Salamanca, Spain \\ ombaf@isep.ipp.pt, mafgh@isep.ipp.pt, $\underline{\operatorname{lufog} @ \text { isep.ipp.pt, pnfar@isep.ipp.pt, zav@isep.ipp.pt, corchado@ usal.es }}$
}

\begin{abstract}
This paper represents an experimental real-time implementation of an incentive-based demand response program with hardware demonstration of a home energy management system. This system controls the electricity consumption of a residential electricity customer. For this purpose, the real consumption and generation profiles of a typical Portuguese household equipped with a home-scale photovoltaic system are employed. These profiles are simulated by the real-time digital simulator using real hardware resources. In the case studies, three different scenarios are simulated for a period of 24 hours with the consideration of the demand response programs and a 2 kW photovoltaic system. Different pricing scenarios are considered and the performance of the home energy management system is evaluated under each scenario. The focus is given to demonstrate how a home-scale photovoltaic system, and demand response programs, especially load-shifting scenario, can be costeffective in the daily electricity costs of the residential customers.
\end{abstract}

Index Terms - Demand Response, Load-Shifting, Real-Time Simulation, Renewable Energy Resources, Financial Profits, Daily Electricity Costs.

\section{INTRODUCTION}

Demand Response (DR) is referred to the deliberate modification of the electricity consumption pattern in order to response to financial incentives provided by the network operator due to the economic or technical reasons [1], [2]. A well-designed DR program decreases the energy consumption as well as energy costs of the end-user during the peak-times [3]. Additionally, if the DR programs merge to the Distributed Generation (DG) resources, which can be implemented via smart grids, the DR concepts would be fully addressed [4].

DR is classified into the two main parts [5]: price-based, and incentive-based. Price-based DR is defined as modification of the electricity consumption profile in order to response to the prices variations. Incentive-based DR is related to the fixed or time varying incentive plans, which are represented to the customers by the Retail Electricity Provider (REP).

On the other hand, REPs have mandate for transmitting the electricity from the local resources to the end-users, and

The present work was done and funded in the scope of the following projects: EUREKA - ITEA2 Project SEAS with project number 12004; H2020 DREAM-GO Project (Marie Sklodowska-Curie grant agreement No 641794); AVIGAE (3401/08/SI/2015); and UID/EEA/00760/2013 funded by FEDER Funds through COMPETE program and by National Funds through FCT. providing the DR programs as well [6]. The REP is the last part of the energy supply chain and can be defined as an intermediary that purchases electricity from the wholesale markets and/or from Distributed Energy Resources (DERs) and sells it to the end-users. This entity determines efficient DR programs to increase their payoff in the market, such as designing financial incentive plans for demand reduction and communicating with the customers one day in advance [3]. Thereupon, the customers can execute these DR programs, based on their capabilities. It is obvious that the household that are not equipped with an intelligent Home Energy Management System (HEMS), cannot easily benefit from these programs. Load-shifting is one of the common forms of the DR programs where the high consumption loads are shifted from the peak periods to the off-peak periods. This scheme is widely used in the residential areas, trying to change the controllable consumption pattern of the domestic loads [7].

Furthermore, by employing the Renewable Energy Resources (RERs), namely home-scale Photovoltaic (PV) systems, and integrating with the implementation of DR programs, the customers are enabled to have more active participation in the electricity markets. This opportunity not only reduces the electricity costs of the customers, but also it decreases the pressure on the transmission grid during the peak times [8]. However, implementation of these scenarios in the current power distribution networks requires several levels of infrastructures, such as Advanced Metering Infrastructure (AMI) and smart metering systems [9].

In this paper, the hardware demonstration presented in [10] is used, and it is assumed that the REP is offering the financial incentive plans developed in [3], [6] to the customers for the desired demand reduction.

In this model, the HEMS controls the consumption of a household that procures the energy needs from the grid and the PV system installed in the building. The customer tries to benefit as much as it can from the DR programs in the form of discounts applied to its monthly electricity bill. Exploiting from the financial compensation depends on the type of the contracts that the customers have made with the REP and the incentive 
plans that the REP offers. The customer can shift the loads that are not time-sensitive in order to receive the financial incentives.

\section{RELATED WORKS}

According to the references, there are similar works that investigate the DR programs in real-time using real data. In [11], the authors represented different scenarios of implementing photovoltaic systems and Li-based batteries under ERCOT's DR program design for a typical Texas residential load. In [12], the authors integrated a home energy simulator and MATLAB TM together for DR development and evaluation from the consumer's perspective. They compared characteristics of different DR strategies and demonstrated how they affected by dynamic pricing tariffs, seasons, and weather.

The main contribution of this paper is to implement, in realtime, the active participation of a typical customer in the electricity markets by employing the incentive-based DR programs and a home-scale PV system. Additionally, financial profits obtained by the customer in a single day are illustrated and compared in the case studies. Since the real data of the consumption and generation are more precise for the calculation, the main approach of this model is to use the data of the real hardware resources, which are integrated in the realtime simulator as Hardware-In-the-Loop (HIL), for the calculation of the financial profits of a typical consumer.

This paper is organized as follows. The incentive-based DR program has been described in the Section III. Section IV represents the HEMS architecture and more details on how it operates. Simulated scenarios and case studies are described in Section V. Finally, the paper concludes with several main points in the section VI.

\section{INCENTIVE-BASED DEMAND RESPONSE PROGRAMS}

DR program is a price-based or incentive-based program or tariff to motivate changes in electricity consumption in response to changes in price [3]. In other words, they aim to alter load consumption in response to generation and transmission conditions, which is reflected on the wholesale electricity prices. The electricity consumption patterns of the electricity users are stimulated with the incentives and/or the prices offered to them. The changes in consumption patterns can lead to peak reduction and lowers the generation capacity requirements [11].

The program operator of an incentive-based DR program offers its' clients fixed or variable financial incentives, in addition to the fixed/Time-Of-Use (TOU) pricing scheme, in order to manage the demand consumption. U.S. Department of energy introduced the following classification for the incentivebased DR programs in 2006 [5], [13]:

- Direct load control: Allowing the DR operator to remotely control the clients' equipment.

- Interruptible/curtailable service: Integrating the curtailment options into the retail contracts with the clients. The customer receives the incentives as rate discounts or bill credits. They might be penalized in some circumstances, especially if they fail to reduce the consumption during contingencies.
- Demand bidding/buyback programs: Offering DR bids by large customers to curtail demand based on wholesale electricity market prices.

- $\quad$ Emergency DR programs: Offering incentives to clients to reduce consumption during reserve shortfalls.

- Capacity market programs: Offering load curtailment by the demand-side as system capacity to replace conventional generation or delivery resources.

REPs can be the program operator in some of the above mechanisms, however, for the rest they do not obtain any benefit through employing the program or they do not have the required infrastructure to apply the program. In this paper, the client responds to the curtailable service provided by the REP.

Employing the incentive-based DR programs is an ideal option for REPs to maximize their short-term profits in the market. These programs also benefit the customers and help them reduce their household energy costs.

The REP offers the financial incentives for each $\mathrm{kWh}$ of demand reduction and notifies them the baseline load consumption to the clients one day in advance. If customers consume energy beneath the baseline during the periods that the REP has set the financial incentives, it receives the incentives for each $\mathrm{kWh}$ of demand reduction.

\section{HOME ENERGy MANAGEMENT SYSTEM}

The system used in this paper includes a $4 \mathrm{kVA}$ load, representing the household consumption and a $2 \mathrm{~kW}$ PV system, installed in the GECAD laboratory at Institute of Engineering - Polytechnic of Porto (ISEP/IPP), Portugal. This system simulates the load profile and the PV generation of a typical household in Portugal. OPAL-RT® real-time simulator acts as a HEMS, which controls the consumption of the $4 \mathrm{kVA}$ load and the PV system through the several standard communication protocols. Fig. 1 demonstrates an overview of the model employed in this paper.

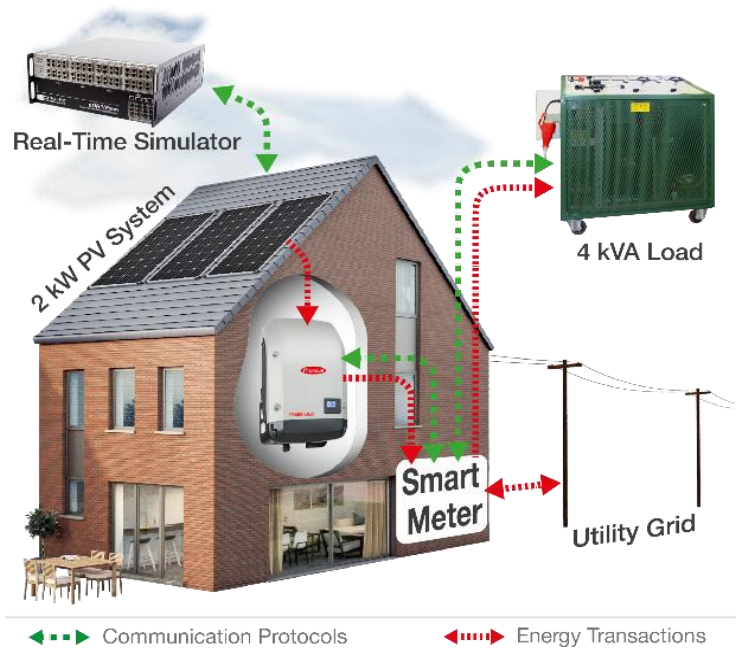

Fig. 1 - An overview of the system.

The consumption profile of the house is simulated by $4 \mathrm{kVA}$ load. An Arduino® (www.arduino.cc) equipped with an Ethernet shield, Relay shield, and an energy meter manages the consumption of the $4 \mathrm{kVA}$ load. The desired power rates are 
transmitted from the OPAL to the $4 \mathrm{kVA}$ load through the Ethernet interface with Modbus TCP/IP protocol, and simultaneously the real-time consumption of the $4 \mathrm{kVA}$ load is sent to the OPAL. By this way, the HEMS employs the $4 \mathrm{kVA}$ load as a HIL [10].

Moreover, the inverter of the $2 \mathrm{~kW}$ PV system sends the real-time generation data to the HEMS via Ethernet interface with Modbus TCP/IP protocol. More detailed information on how the HEMS controls the $4 \mathrm{kVA}$ load and the PV system is available on [10].

The tariffs and the rules of the incumbent Portuguese electricity retailer are applied in this simulation [14]. In Portugal, the retailers have started offering different types of DR programs to their clients. This process has been accelerated after phasing out the regulated retail rates in Portugal. The residential customer in this model is willing to shift the loads in order to gain financial benefits from the DR programs offered by its' REP.

The consumption data used in this paper is driven from [15]. Therefore, the historical consumption profile of the private house 3 on the date of 24/01/2013 for 24 hours (from 00:00 AM to $23: 59 \mathrm{PM}$ ) was chosen for the case studies. The interval time between the two measured samples is one minute.

The PV generation curve used in this model is the real production data of the GECAD's PV system on 16/10/2015. The generation data in this date was compatible with the historical consumption profile of the house. These curves are shown in the Fig. 2. It should mention that the model has the capability of consuming its own produced energy and injecting the additional power into the utility grid.

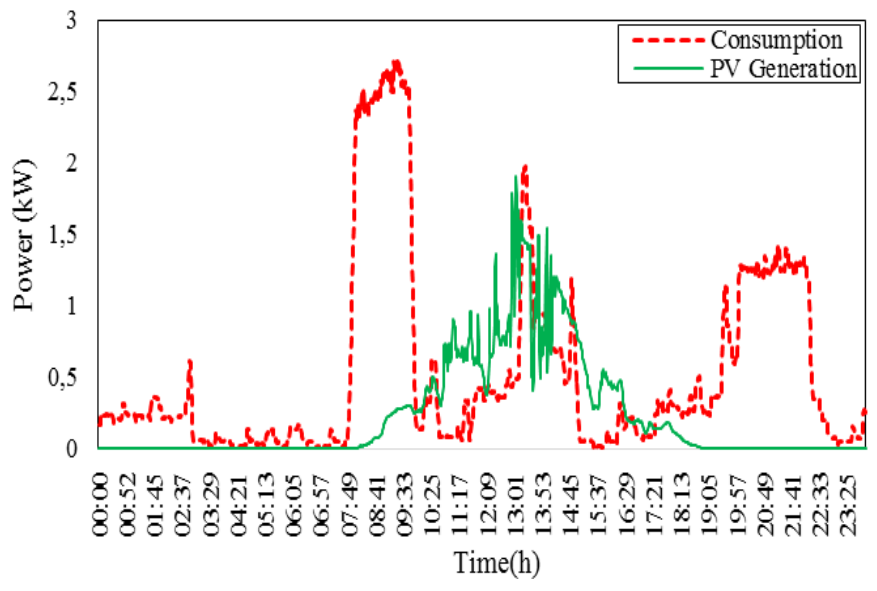

Fig. 2 - Daily consumption and generation profiles of a typical house in Portugal.

In the next parts, this system will be employed for a realtime implementation of an incentive-based DR program, and demonstration of the active participation of the end-users in the electricity markets.

\section{SimUlated SCENARIOS}

Three different scenarios are simulated in this paper and compared from the point of view of the customers' daily financial benefits obtained from participating in the relevant pricing scheme or the DR program.
Financial incentive plans used in this paper are based on the incentive-based DR programs developed in [3], [6]. These plans have been designed through a systematic approach for REPs in short-terms markets to encourage the customers to shift their load and change their consumption patterns [3], [6]. The incentive plans are shown in Table I.

TABLE I. INCENTIVE-BASED DR PROGRAM.

\begin{tabular}{cccccc}
\hline Hour & $\begin{array}{c}\text { Incentive } \\
(\boldsymbol{E} \boldsymbol{R} / \boldsymbol{k} \boldsymbol{W h})\end{array}$ & $\begin{array}{c}\text { Baseline } \\
\text { load } \\
(\boldsymbol{k W h})\end{array}$ & Hour & $\begin{array}{c}\text { Incentive } \\
(\boldsymbol{E U R} / \boldsymbol{k W h})\end{array}$ & $\begin{array}{c}\text { Baseline } \\
\text { load } \\
(\boldsymbol{k W h})\end{array}$ \\
\hline 1 & 0 & 0 & 13 & 0.032 & 0.32 \\
2 & 0 & 0 & 14 & 0.044 & 0.5 \\
3 & 0 & 0 & 15 & 0.037 & 0.5 \\
4 & 0 & 0 & 16 & 0.036 & 0.38 \\
5 & 0 & 0 & 17 & 0.030 & 0.1 \\
6 & 0 & 0 & 18 & 0.022 & 0.15 \\
7 & 0.042 & 0.02 & 19 & 0.019 & 0.22 \\
8 & 0.036 & 0.1 & 20 & 0.031 & 0.46 \\
9 & 0.054 & 1.9 & 21 & 0.008 & 1 \\
10 & 0.021 & 1.85 & 22 & 0.012 & 0.9 \\
11 & 0.052 & 0.15 & 23 & 0.016 & 0.4 \\
12 & 0.031 & 0.1 & 24 & 0 & 0 \\
\hline
\end{tabular}

It is assumed that the incentive plans for the desired demand reduction for each day are sent to customers the night before, and if the customers decrease their consumption below the baseline load, they will receive the financial incentives from the REP.

Moreover, based on the article 24 of the [16], and Portuguese system of the Iberian electricity market [17], the selling price of the PV energy produced by the end-users in the date of $16 / 10 / 2015$ is EUR 0.0522 per $\mathrm{kWh}$.

In the next parts of this section, the real data will be employed in order to calculate the daily electricity cost of a typical Portuguese house equipped with a HEMS under different scenarios.

\section{A. Case Study 1}

In this case study the basic scenario is modeled in order to be used for comparison with the next two scenarios. The household in this case has no capability of executing DR programs, and also no PV system is installed on the house. The per-minute consumption curve shown in Fig. 2 is applied in this case study. The purpose is to obtain the daily electricity cost of this customer under the typical circumstance and compared with the final results of the next case studies. Therefore, the daily electricity cost $\left(C_{C S 1}\right)$ is calculated by

$$
\begin{aligned}
& C_{C S 1}=\sum_{h=1}^{24} E_{\text {Load }}(h) \times \operatorname{Pr}(h) \\
& E_{\text {Load }}(h)=\frac{\sum_{i=1}^{60} P_{\text {Load }}(i+(h-1) \times 60)}{60}, \quad \forall \mathrm{h}=1, \ldots, 24
\end{aligned}
$$

Where $P_{\text {Load }}$ signifies the per-minute load consumption in $\mathrm{kW}$, which is demonstrated on Fig. $2, E_{\text {Load }}(h)$ represents the energy consumed at each hour in $\mathrm{kWh}$ that has been shown on Fig. 3, and $\operatorname{Pr}(h)$ stands for the hourly electricity price in EUR/kWh. Therefore, the daily electricity cost of this single day is EUR 2.3020. In the next case studies, the customer efforts to minimize this energy procurement cost. 


\section{B. Case Study 2}

In this section, the capability of executing DR programs is included on the system while no PV system is considered.

The customer applies the DR programs through the loadshifting scenario. This process is done according to the baseline consumption pattern demonstrated in Table I. Fig. 3 illustrates the shifted consumption curve of this residential load.

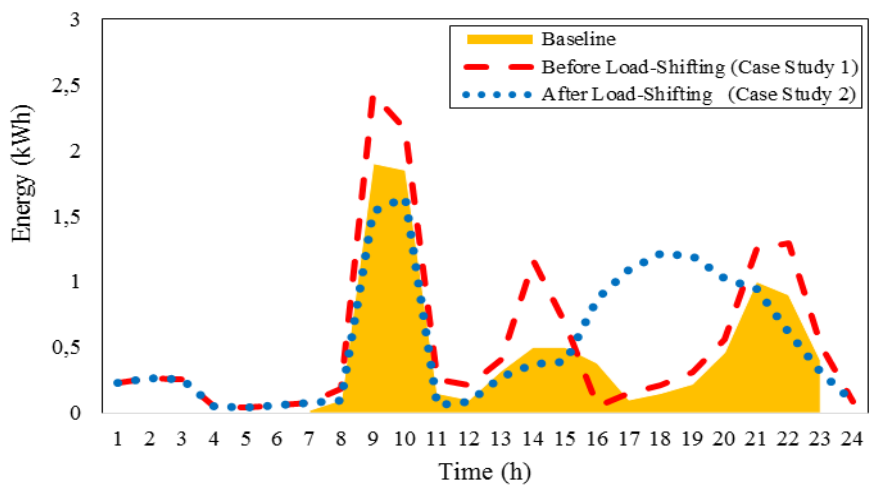

Fig. 3 - Consumption curve after applying load-shifting.

One of the important points of the load-shifting scenario is to have a same amount of the energy consumption at the end of the day, even if the peak demand is shaved and shifted to the off-peak periods. By the same token, for proving the correctness of the load-shifting scenario executed in the model, the following equation is used:

$$
\begin{aligned}
& E_{\text {Total }}(i)=\frac{P_{\text {Load }}(i)}{60}+E_{\text {Total }}(i-1), \quad \forall \mathrm{i}=1, \ldots, 1440 \\
& E_{\text {Total }}(0)=0
\end{aligned}
$$

Where $E_{\text {Total }}(i)$ is the total energy consumed in the end of time period of $(i)$ in $\mathrm{kWh}$. The interval time between the two values of $(i)$ is one minute. By this way, $E_{\text {Total }}(t)$, which is the total energy consumed in 24 hours, before the implementation of load-shifting was $12.889 \mathrm{kWh}$, and after that is $12.768 \mathrm{kWh}$. As you can see, there is not much difference between these two values, therefore, the load-shifting process is successfully executed in this case study.

By regarding to this load-shifting and the related DR programs, the daily electricity $\operatorname{cost} C_{C S 2}$ can be calculated by

$$
\begin{aligned}
& C_{C S 2}=C_{1}-C_{2} \\
& C_{1}=\sum_{h=1}^{24}\left(E_{\text {Load }}(h)-E_{D R}(h)\right) \times \operatorname{Pr}(h) \\
& C_{2}=\sum_{h=1}^{24} E_{D R}(h) \times \operatorname{Pr}_{D R}(h)
\end{aligned}
$$

Where $E_{D R}(h)$ represents the energy reduction during each hour in $\mathrm{kWh}$, and $\operatorname{Pr}_{D R}(h)$ denotes the incentive paid to the customer for each $\mathrm{kWh}$ of $E_{D R}(h)$ energy reduction in EUR/kWh. Therefore, the daily electricity cost of this case study is EUR 2.2350. Compared to the case study 1, the customer achieved $3 \%$ financial profit and its daily electricity cost reduced EUR 0.067 at the end of this single day. As you can see, by execution of such DR programs, not only the customers attain to the financial profits, but also they assist to improve the performance of the utility grid in the peak-times.

\section{Case Study 3}

This case study is referred to the same house with consideration of DR programs and the $2 \mathrm{~kW}$ PV system. For this purpose, the consumption and generation curves illustrated in the Fig. 2 have been used. Additionally, the incentive plan and the baseline used in the case study 2 have been employed. The daily electricity cost of this case $C_{C S 3}$ is

$$
\begin{aligned}
& C_{C S 3}=C_{B u y}-C_{S e l l}-C_{D R} \\
& \begin{cases}C_{\text {Buy }}=\sum_{h=1}^{24}\left(E_{\text {Load }}(h)-E_{D R}(h)-E_{P V}(h)\right) \times \operatorname{Pr}(h) & E_{\text {Load }}>E_{P V} \\
C_{\text {Sell }}=\sum_{h=1}^{24}\left(E_{P V}(h)-E_{\text {Load }}(h)-E_{D R}(h)\right) \times P r_{P V}(h) & E_{\text {Load }}<E_{P V}\end{cases} \\
& E_{P V}(h)=\frac{\sum_{i=1}^{60} P_{P V}(i+(h-1) \times 60)}{60}, \quad \forall \mathrm{h}=1, \ldots, 24
\end{aligned}
$$

Where $C_{B u y}$ is the total electricity cost of the energy purchased from the utility grid, $C_{\text {Sell }}$ is the total cost of the energy sold to the utility grid, and $C_{D R}$ represents the incentive paid to the customer for the demand reduction, which is equal to the equation (6). Also $P_{P V}(i)$ denotes the per-minute PV generation in $\mathrm{kW}$ shown on Fig. $2, E_{P V}(h)$ signifies the produced energy by the PV at each hour in kWh shown on Fig. 4, and $\operatorname{Pr}_{P V}(h)$ stands for the price of selling the PV energy, which is $0.0522 \mathrm{EUR} / \mathrm{kWh}$.

In this case, while the energy demand of the house is not provided with the PV production, the customer purchases energy from the utility grid. In the reverse mode, while the PV production is more than the energy demand, not only it supplies the local loads, but also the excess of the produced energy will be injected to the utility grid. This scenario enables the customer to have active participation in the electricity markets. Fig. 4 demonstrates the implementation of the load-shifting scenario according to the related DR program and the PV generation.

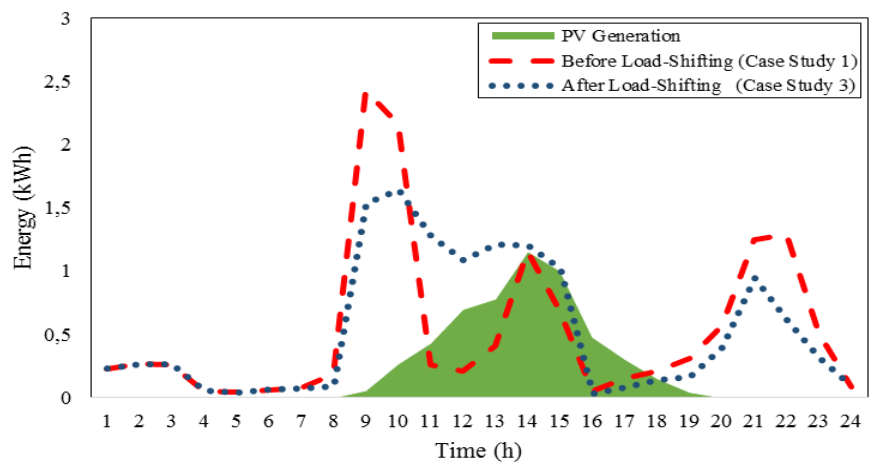

Fig. 4 - Applying load-shifting scenario in the consumption profile based on the PV generation.

As it is clear in the Fig. 4, the consumption has been shifted to the high PV generation moments. Since the customer consumes its own produced energy, the purchased energy from 
the utility grid will be reduced. Moreover, while the consumption is less than the PV generation and also is situated beneath the baseline load provided by REP, not only the customers sell the PV production to the utility grid, but also they receive the financial incentives from the REP for the desired demand reduction. By this way, the customers achieve to the maximum financial profits.

Therefore, in this case, $C_{B u y}$ is EUR 1.4212, $C_{\text {Sell }}$ is EUR $0.0354, C_{D R}$ is EUR 0.0997, and the total daily electricity cost $C_{C S 3}$ is EUR 1.2860. Compared to the case study 1, the customer achieved $44 \%$ cost reduction. These results prove that how a home-scale PV system can be cost-effective for the customers.

\section{Results}

Final results of the three case studies implemented in this paper are demonstrated in the Table II. These results illustrate the financial profits that the customer obtained in a single day by executing the DR programs and a home-scale RERs.

TABLE II. FINANCIAL COMPARISON OF THE RESULTS.

\begin{tabular}{cccc}
\hline $\begin{array}{c}\text { Case } \\
\text { Study }\end{array}$ & Features & $\begin{array}{c}\text { Daily Cost } \\
(\text { EUR })\end{array}$ & $\begin{array}{c}\text { Cost-Reduction } \\
(\text { Compared to C CS 1 }\end{array}$ \\
\hline 1 & $\begin{array}{c}\text { No DR Program } \\
\text { No PV system }\end{array}$ & 2.3020 & - \\
2 & $\begin{array}{c}\text { DR Program } \\
\text { No PV system } \\
\text { DR Program } \\
\text { PV System }\end{array}$ & 2.2350 & $3 \%$ \\
3 & 1.2860 & $44 \%$ \\
\hline
\end{tabular}

As it is clear in the Table II, the financial benefits are completely depend on the capabilities of the customers. By participation in the DR programs and installation of the homescale RERs, they can obtain a significant amount of financial profits and electricity cost-reduction.

\section{CONCLUSIONS}

Implementing demand response programs at the residential level benefits all sides of the market. It benefits the customers by lowering their electricity bills and the retailers by reducing their cost at wholesale market by shifting the load to lower prices periods. Furthermore, a well-designed demand response program shifts some parts of demand to off-peak periods and relieves the grid congestion. This scenario assists to decrease the requirements of peaking generation capacity by reducing over-generation problems during the night.

The model implemented in this paper proposed a real-time simulation of an incentive-based demand response program for a residential customer. Real consumption and generation profiles were used in this simulation. Additionally, the active participation of a typical customer in the electricity markets was demonstrated by considering a home-scale photovoltaic system. Financial profits obtained by this customer in a single day were demonstrated and compared in the case studies. The results of case studies illustrate that how a home-scale photovoltaic system and demand response execution, especially load-shifting scenario, are cost-effective for the customers. However, these financial profits are completely depend on the capabilities of the end-users.

In the future work, this model will be executed not only for a single day, but also for more days in order to gain more precise results.

\section{REFERENCES}

[1] N. Mahmoudi, M. Eghbal, and T. Saha, "Employing demand response in energy procurement plans of electricity retailers," International Journal of Electrical Power \& Energy Systems, vol. 63, pp. 455-460, 2014.

[2] P. Faria, Z. Vale, and J. Baptista, "Demand Response Programs Design and Use Considering Intensive Penetration of Distributed Generation," Energies, vol. 8, no. 6, pp. 6230-6246, 2015.

[3] M. Fotouhi Ghazvini, P. Faria, S. Ramos, H. Morais, and Z. Vale, "Incentive-based demand response programs designed by asset-light retail electricity providers for the day-ahead market," Energy, vol. 82, pp. 786-799, 2015.

[4] X. Fang, S. Misra, G. Xue, and D. Yang, "Smart Grid - The New and Improved Power Grid: A Survey," IEEE Communications Surveys \& Tutorials, vol. 14, no. 4, pp. 944-980, 2012.

[5] P. Faria, and Z. Vale, "Demand response in electrical energy supply: An optimal real time pricing approach," Energy, vol. 36, no. 8, pp. 53745384, 2011.

[6] M. Fotouhi Ghazvini, J. Soares, N. Horta, R. Neves, R. Castro, and Z. Vale, "A multi-objective model for scheduling of short-term incentivebased demand response programs offered by electricity retailers," Applied Energy, vol. 151, pp. 102-118, 2015.

[7] M. Vlot, J. Knigge, and J. Slootweg, "Economical Regulation Power Through Load Shifting With Smart Energy Appliances," IEEE Transactions on Smart Grid, vol. 4, no. 3, pp. 1705-1712, 2013.

[8] L. Gomes, P. Faria, H. Morais, Z. Vale, and C. Ramos, "Distributed, Agent-Based Intelligent System for Demand Response Program Simulation in Smart Grids," Intelligent Systems, IEEE, vol. 29, no. 1, pp. 56-65, 2014.

[9] R. Walawalkar, S. Fernands, N. Thakur and K. Chevva, "Evolution and current status of demand response (DR) in electricity markets: Insights from PJM and NYISO," Energy, vol. 35, no. 4, pp. 1553-1560, 2010.

[10] O. Abrishambaf, L. Gomes, P. Faria, and Z. Vale, "Simulation and Control of Consumption and Generation of Hardware Resources in Microgrid Real-Time Digital Simulator," in 2015 IEEE PES Innovative Smart Grid Technologies Latin America (ISGT LATAM), Montevideo, Uruguay, 5-7 Oct. 2015, pp. 863-868.

[11] M. Liu, W. Lee, and L. Lee, "Financial Opportunities by Implementing Renewable Sources and Storage Devices for Households Under ERCOT Demand Response Programs Design," IEEE Transactions on Industry Applications, vol. 50, no. 4, pp. 2780-2787, 2014.

[12] S. Li, D. Zhang, A. Roget, and Z. O'Neill, "Integrating Home Energy Simulation and Dynamic Electricity Price for Demand Response Study," IEEE Transactions on Smart Grid, vol. 5, no. 2, pp. 779-788, 2014.

[13] Office of Electricity Delivery and Energy Reliability, "Benefits of Demand Response in Electricity Markets and Recommendations for Achieving them," U.S Department of Energy, 2006.

[14] Edp.pt, "EDP," 2015. [Online]. Available: http://www.edp.pt. [Accessed: 04- Nov- 2015].

[15] Sites.ieee.org, "Data Sets «IEEE Intelligent Data Mining and Analysis (IDMA)," 2015. [Online]. Available: http://sites.ieee.org/psaceidma/data-set. [Accessed: 04- Nov- 2015].

[16] Ministério do Ambiente, Ornamento do Território e Energia, 'DecretoLei n. ${ }^{\circ}$ 153/2014', Diário da República n. ${ }^{\circ}$ 202, 20 October 2014.

[17] Omie.es, "Iberian Electricity Market," 2015. [Online]. Available: http://www.omie.es/files/flash/ResultadosMercado.swf. [Accessed: 05Nov- 2015]. 